\title{
Process-based allometry describes the influence of management on orchard tree aboveground architecture
}

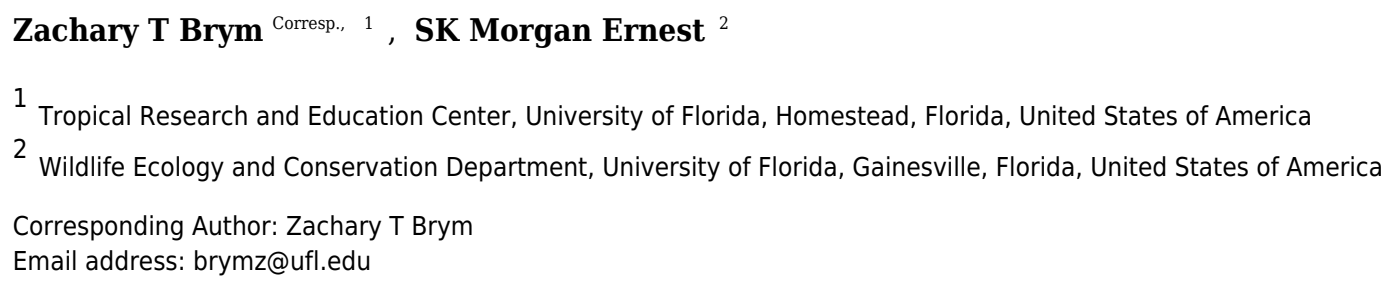

We evaluated allometric relationships in length, diameter, and mass of branches for two variably managed orchard tree species (tart cherry, Prunus cerasus; apple, Malus spp.). The empirically estimated allometric exponents $(a)$ of the orchard trees were described in the context of two processed-based allometry models that make predictions for $a$ : the West, Brown and Enquist fractal branching model (WBE) and the recently introduced Flow Similarity model (FS). These allometric models make predictions about relationships in plant morphology (e.g. branch mass, diameter, length, volume, surface area) based on constraints imposed on plant growth by physical and physiological processes. We compared our empirical estimates of a to the model predictions to interpret the physiological implications of pruning and management in orchard systems. Our study found strong allometric relationships among the species and individuals studied with limited agreement with the expectations of either model. The 8/3-power law prediction of the mass $\sim$ diameter relationship by the WBE, indicative of biomechanical limitations, was marginally supported by this study. Length-including allometric relationships deviated from predictions of both models, but shift toward the expectation of flow similarity. In this way, managed orchard trees deviated from strict adherence to the idealized expectations of the models, but still fall within the range of model expectations in many cases despite intensive management. 
1 Process-based allometry describes the influence of management on orchard

2 tree aboveground architecture

3

4 Zachary T. Brym¹, S.K. Morgan Ernest ${ }^{2}$

5

$6{ }^{1}$ Tropical Research and Education Center, University of Florida, Homestead, FL, USA

$7 \quad 2$ Wildlife Ecology and Conservation Department, University of Florida, Gainesville, FL, USA

8

9 Corresponding Author:

10 Zachary Brym ${ }^{1}$

11

12 Email Address: brymz@ufl.edu 
13 Abstract We evaluated allometric relationships in length, diameter, and mass of branches

14 for two variably managed orchard tree species (tart cherry, Prunus cerasus; apple, Malus spp.).

15 The empirically estimated allometric exponents $(a)$ of the orchard trees were described in the

16 context of two processed-based allometry models that make predictions for $a$ : the West, Brown

17 and Enquist fractal branching model (WBE) and the recently introduced Flow Similarity model

18 (FS). These allometric models make predictions about relationships in plant morphology (e.g.

19 branch mass, diameter, length, volume, surface area) based on constraints imposed on plant

20 growth by physical and physiological processes. We compared our empirical estimates of $a$ to

21 the model predictions to interpret the physiological implications of pruning and management in

22 orchard systems. Our study found strong allometric relationships among the species and

23 individuals studied with limited agreement with the expectations of either model. The 8/3-power

24 law prediction of the mass $\sim$ diameter relationship by the WBE, indicative of biomechanical

25 limitations, was marginally supported by this study. Length-including allometric relationships

26 deviated from predictions of both models, but shift toward the expectation of flow similarity. In

27 this way, managed orchard trees deviated from strict adherence to the idealized expectations of

28 the models, but still fall within the range of model expectations in many cases despite intensive

29 management. 


\section{Introduction}

32 The physical structure of a plant emerges from species-specific growth strategies for accessing

33 scarce environmental resources such as light, water, and nutrients (Pacala and Tilman 1994;

34 Grossman and DeJong 1995; Kobe 2006). While species vary in their strategies for growing in

35 resource-limited environments, even when resources are not limited, growth is constrained by

36 physiological limits on processes such as photosynthesis and resource transport (Murneek and

37 Logan 1932; Niklas and Kirchner 1984). The diversity of plant form that exists in nature reveals

38 the many ways that plants evolved to balance trade-offs between external environmental and

39 internal physiological limitations (Niklas 1997). Despite the diversity of plant form, however,

40 there are still emergent patterns in plant structure that may reflect the impact of underlying

41 constraints on plant physiology (Price et al. 2010).

42 One aspect of plant form where physiological constraints on plant growth may be evident

43 is aboveground morphology or architecture (Niklas 2004). Aboveground morphology of plants

44 often exhibits regular patterns referred to as allometric relationships (Huxley and Teissier, 1936;

45 Lacointe 2000). Allometric relationships describe how plant size (e.g., mass or diameter) relates

46 to other dimensions of morphology such as branch length, surface area, or volume. These

47 relationships are often highly constrained (i.e., show limited variance) and are typically well

48 described by power law equations of the form:

$49=b x^{\mathrm{a}}$

50 where $y$ is the measurement of some trait of interest, $x$ is a measure of plant size, and $a$ and $b$

51 represent values that describe the form of the relationship, the allometric exponent and

52 multiplier, respectively. This equation is often expressed in log-log space which then expresses $a$

53 as the slope of a linear relationship and $b$ as the $y$-intercept. 
Although powerful for generalizing patterns in plant architecture, allometries are often

55

established for specific species of plants grown in specific conditions (Brown, 1997, Niklas 2004). Various models exist attempting to explain why allometries often take the general form of a power law and why we often see a narrow range of allometric exponent values $(a)$ in empirical data. The exact value and importance of $a$ remains debated (Thompson, 1942; Enquist et al. 2007; Coomes et al. 2011; Price et al. 2012) as is the usefulness of an allometric approach to describing plant form, especially in applied settings (Le Roux et al. 2001). Applied models of plant architecture require detailed information for plant response to growing conditions and management (Le Roux et al. 2001; Lang and Lang 2008). Therefore, the future improvement of morphological plant modeling requires availability of detailed information of plant growth and management linked with models that provide detailed plant information with mathematical perspective (Bucksch et al. 2017).

This study contributes detailed information about plant morphology for mature orchard fruit species and links the physiological information with two process-based plant allometry models that provide expected ranges of allometric exponent values that should be consistent across the diversity of plant species. However, orchard trees experience unique growing conditions that may be expected to deviate from the general patterns described in the processbased plant allometry models. The genetic material and management regimen of orchard trees provides a distinct manipulated environment and set of growing conditions. Clonal rootstocks that influence tree growth and partitioning are grafted to scion wood that produces favorable fruit (Robinson 2007). The orchard environment is heavily subsidized with water and nutrients, reducing the effect of resource limitation on plant architecture. With high subsidies of water and nutrients, physiological constraints should be the primary influence on plant growth (Deng et al. 
77 2012). However, tree architecture is also directly manipulated by pruning and training to

78 improve light penetration, airflow, and fruit production (Lauri et al. 2011). Dormant season

79 pruning, the destructive removal of branches in winter months, impacts the growth trajectory of

80 trees by removing growing nodes and displacing growth hormones. Trees respond to pruning the

81 following growing season with localized invigoration of retained buds and branches and an

82 overall dwarfing of tree size (Ferree and Schupp 2003). How controlled genetic material,

83 environmental manipulation, and pruning interact to affect the overall allometry of an orchard

84 tree is unknown.

85 We compare two variably managed orchard tree species using process-based allometry models as context to describe the effects of human manipulation on allometry. Allometries have

87 been widely used in the management of orchard systems to predict carbon partitioning to fruit 88 (Westwood and Roberts 1970; Lacointe 2000). However, the allometric relationships used in

89 horticulture can be rigid and lack linkages to the underlying processes generating estimates of $a$.

90 In contrast, a recently proposed process-based allometry model called Flow Similarity (FS)

91 attempts to explicitly explain the variation in $a$ by incorporating two fundamental physical

92 processes constraining plant growth: hydraulics and biomechanics (Price et al. preprint). The

93 hydraulic constraint is described as 'flow similarity', which is the condition where a constant

94 flow rate and velocity of water is maintained through the plant vascular network by area-

95 preserving branching (Shinozaki et al. 1964; McCulloh and Sperry 2005). The biomechanical

96 constraint is described as 'elastic similarity', where each branch grows to the structural limit at

97 which if it were to grow any larger it would break under its own weight (McMahon and

98 Kronauer 1976; Niklas 1992). The FS model explicitly recognizes that a tree grows dynamically

99 in order to optimize water use while providing a sufficient structural architecture. New growth 
100 and branches at the distal end of a plant are likely to express flow similarity, while the trunk and

101 basal structural branches that bear the majority of weight of the plant are likely to express elastic

102 similarity. From this dynamic view of interacting physical constraints, FS predicts a range of $a$

103 that falls within the bounds of hydraulic and biomechanical constraints (Table 1). The FS

104 approach to expressing dynamic constraints between hydraulic and biomechanical limits is

105 included as context for the interpretation of our analysis in addition to a more established model

106 derived by West, Brown and Enquist that is built on similar processes but makes different

107 predictions (WBE; 1997). WBE assumes that the interaction between biomechanical and

108 hydraulic processes is fixed within and across species, but still offers a range of $a$ predictions in

109 some cases that recognizes the various physiological constraints between small and young

110 growth that is constrained by water versus large and old growth that is constrained by weight

111 (Table 1; Enquist et al. 2007).

112 The application of process-based allometry models to interpret the plant architecture of

113 orchard trees provides the opportunity to understand how human management impacts the

114 fundamental physiological constraints described by allometry and how these constraints on plant

115 growth and morphology influence how managed trees respond to human manipulation. Are the

116 allometric relationships of managed trees still consistent with expectations from process-based

117 allometric models built to explain plant architecture of unmanaged trees? Or, does human

118 manipulation of the natural architecture push orchard trees away from basic physical and

119 biological constraints to exhibit forms with little comparison in unmanaged systems?

\section{Materials \& Methods}


123 System

124 Two Rosaceous species from experimental orchard blocks at the Utah State University

125 Kaysville Research Farm (2011-2013) in Davis County, Utah were used in this study: tart cherry

126 (Prunus cerasus) and apple (Malus spp.). The production systems sampled for tart cherry and

127 apple differ in management intensity and genetic complexity.

128 The sampled tart cherry orchard block was twenty-four years-old, near the end

129 of peak production age for similar orchard systems. Individuals consisted of a clonal scion

130 (cultivar: 'Montmorency') grafted on to closely related seedling rootstocks (Prunus mahaleb).

131 These individuals are described generally by vigorous growth and wide crotch angles $\left(57.1^{\mathrm{O}} \pm\right.$

132 27.9). Fruit-bearing spurs, stubby twigs that grow off of main branches, tend to be located on the

133 proximal two-thirds of parent branches (Maguylo et al. 2004). A multiple leader 'open-vase'

134 canopy was developed in the first few years of growth by selecting three to five main structural

135 branches for ideal orientation and branching angle. Following initial canopy development,

136 individuals received relatively minor annual pruning, $\sim 10 \%$ total biomass, to improve light

137 penetration, air flow, and fruit set. No pruning occurred for five years prior to the study.

138 The apple block was ten years-old and part of the NC-140 Regional Rootstock Research

139 Project - 2003 'Golden Delicious' Trial (Marini et al. 2014). The individual trees consisted of

140 clonal scions (cultivar: 'Golden Delicious') grafted on to several clonal rootstocks ('Budagovsky

141 9', 'Geneva ${ }^{\circledR} 41$ ', 'Geneva ${ }^{\circledR} 210$ ', 'Malling 26', 'Japan-Morioka 8', 'Pi-AU 56-83'). The

142 'Golden Delicious' cultivar is described as moderately vigorous with wide crotch angles $\left(56.4^{\mathrm{O}} \pm\right.$

143 36.6) and bears fruit on spurs or terminals of short shoots (Ferree and Schupp 2003). Rootstocks

144 primarily drive growth potential and disease resistance and provide a gradient in tree size,

145 introduced from smallest to largest. Budagovsky 9 (B.9) is highly dwarfing, highly productive, 
146 and winter hardy (Budagovsky 1974; Stehr 2007; Hoover et al. 2011). Geneva ${ }^{\circledR} 41$ (G.41) is a

147 dwarf rootstock with wide crotch angles that expresses good yield and fruit size, disease

148 resistance, and winter hardiness (Robinson and Hoying 2004; Fazio et al. 2013). Geneva ${ }^{\circledR} 210$

149 (G.210) is a semi-dwarf with wide crotch angles that is disease resistant, free standing,

150 precocious and productive (Fazio et al. 2013). Malling 26 (M.26) is one of the most common

151 dwarf rootstocks in commercial planting but is susceptible to disease and winter freezing

152 (Hoover et al. 2011; Marini et al. 2009; Robinson et al. 2007). In the western United States it

153 grows more like a semi-dwarf, as observed in our study as an intermediate-sized rootstock.

154 Japan-Morioka 8 (JM.8) is reported as a dwarfing rootstock that is disease resistant; however, it

155 also expresses a semi-dwarf size in some environments, as we observe in our study (Marini et al.

156 2009; Soejima et al. 2010). Pi-AU 56-83 is reported as a dwarfing rootstock from trials in

157 Germany (Fischer 2001), but other reports suggest it expresses as a semi-dwarf with high

158 survival, vigor and fruit weight, but low production (Marini et al. 2009). All apple trees were

159 trained, pruned, and managed consistently according to NC-140 protocols (www.nc140.org).

160 Individuals were trellised and pruned heavily each year, $\sim 25 \%$ total biomass, to maintain one

161 dominant central trunk, or single leader, and whorled terraces of lateral branches for bearing

162 fruit.

163

164 Data Collection

165 We sampled five tart cherry trees for a total of 449 branches and 19 apple trees for a total 166 of 375 branches. The five tart cherries were chosen from a stratified random sample of over 300

167 available trees in the block while excluding senescent or diseased individuals. Six apple

168 rootstocks were chosen from the experimental block to represent a gradient in tree size and 
169 superior survivorship. Three to four individuals of each rootstock were then chosen randomly,

170 except for the industry standard rootstock, M.26, which only had one individual surviving. For

171 each individual sampled, all branches were identified and measured for diameter and length.

172 Branches were defined as a continuous stem between two branching nodes, while twigs were

173 defined as stems supporting only buds or short fruit-bearing stems less than two centimeters in

174 diameter. Branches and twigs were removed from the tree and dried in a large oven for a

175 minimum of one week at $65^{\circ} \mathrm{C}$ and weighed for biomass. Twig length and biomass

176 measurements were limited to one random individual for cherry and each apple rootstock.

177 Sampling occurred at least one full growing season following the last pruning event.

178

179 Branch Classification

180 Branch morphology was classified in three ways to explore allometric patterns below the

181 individual-level: segment, path and subtree (Figure 1). Segment values are the data gathered

182 directly for each branch. Segment length, for instance, is the distance between the proximal end

183 of the branch at one branching node and its distal end at the next branching node. Path values are

184 the data gathered for a given branch and the longest continuous length of distil branches. Path

185 values are the unit of branch length most closely associated with the predictions of WBE (Smith

186 et al 2013). Subtree values are the diameter of a given branch and the total length or mass of that

187 branch and all distil branches. Subtree values are a unit of branch classification associated with

188 FS. The multi-dimensional morphological characteristics, surface area ( $\pi *$ diameter $*$ length)

189 and volume $\left(\pi *(\text { diameter } / 2)^{2} *\right.$ length $)$, are calculated at a segment level with path and subtree

190 level values generated as the appropriate sum of segment level calculations.

191 
192

193

194

195

196

197

198

199

200

201

202

203

204

205

206

207

208

209

211

8

210 Results

212 Branch-level allometries for all branches collected for each species are reported for the

213 length $\sim$ diameter and mass $\sim$ diameter relationships (Table 2). The allometries represent 214 empirical estimates of plant architecture for mature cherry and apple trees. All but five estimated

\section{Data Analysis}

To estimate $a, \log$-log transformed linear relationships for combinations of

morphological characteristics were evaluated using reduced major axis regression (Warton et al. 2006). For each pairwise relationship and branch classification, an estimated $a$ with $95 \%$ confidence intervals and an $r^{2}$ value were determined using the 'SMATR' package in R: (R version 3.1.1). Estimates of $a$ were evaluated by aggregating all branches at the individual and species level.

Because these relationships are not always linear on log-log plots, we also examined whether polynomial fits to the data performed better (Niklas and Hammond 2014). Polynomial fits were tested against linear fits by comparing the AICc values, but did not strongly alter the analysis. Only results of the linear models are reported in the paper.

The addition of twig lengths and mass to branch-level calculations were evaluated among the subset of individuals with the extra sampling effort, with only minor shifts in estimated $a$. Results of this supplemental analysis are not presented in the paper.

Data, programming code, and supplemental information for this analysis can be found freely available online at github.com/weecology/branch-arch/GeneralAllometry.

\section{Allometries}


$21595 \%$ confidence intervals of estimated $a$ at the species level for path and subtree branch

216 classifications overlap and are therefore interpreted as statistically indistinguishable (Table 2;

217 Figure 2). Apple and cherry trees differ in their estimated $a$ at the path level for length $\sim$ volume

218 (Figure 2D), length $\sim$ area (Figure 2F) and mass $\sim$ volume (Figure 2I). Apple and cherry trees

219 differ in their estimated $a$ at the subtree level for surface area $\sim$ volume (Figure 2B) and mass $\sim$

220 volume (Figure 2I).

221

222 Branch classification

223 Across all morphological characteristics, allometric relationships at the path and subtree

224 levels tended to have equal or higher $r^{2}$ values relative to the same relationship calculated at the

225 segment-level (Table 2, Figure 2). Allometric relationships of multi-dimensional morphological

226 characteristics, like surface area and volume, tend to exhibit higher $r^{2}$ values than allometric

227 relationships using linear morphological characteristics, like length and diameter (Figure 2).

228

229

Model expectations

230 When compared to the predictions of the FS model, five of six species-level $a$ estimations

231 at the path level and four of six at the subtree level fall within the expected ranges described by

232 flow similarity and elastic similarity constraints (Figure 2). The relationship that does not fall

233 within the expected range at the path level is diameter $\sim$ volume (Figure $2 \mathrm{C}$ ), while the two

234 relationships that do not fall within the expected range at the subtree level are length $\sim$ volume

235 (Figure 2D) and length $\sim$ surface area (Figure 2F). Only one set of species-level $a$ estimations at

236 the segment level (length $\sim$ volume) narrowly falls within the expected range of FS for both

237 species (Figure 2D). Estimated $a$ tend to shift among segment, path and subtree level branch 
238 classification in the direction towards the flow similarity expectation of FS. At a species level,

239 the path and subtree level mass $\sim$ diameter relationships express a very strong relationship with

240 an estimated $a$ close to the expected value from WBE (Figure 2H). The subtree level relationship

241 confidence intervals overlap with the empirical estimation of 2.53 by Brown (1997; Table 2).

242 The length $\sim$ mass relationship estimated $a$ at a species level appears to deviate significantly

243 from the WBE prediction (Figure $2 \mathrm{G}$ ).

\section{Discussion}

247 Our study describes the allometry of two variably managed orchard tree species drawing context

248 from existing process-based allometry models (Table 2). The allometric relationships of the

249 sampled orchard trees are broadly consistent with each other and the expectations of the process-

250 based allometry models. We find overlap in $95 \%$ confidence intervals of estimated $a$ for tart

251 cherry and apple for most of the allometric relationships evaluated at the path-level (6/9) and

252 subtree-level (7/9) indicating a consistent pattern in growth and resulting tree architecture

253 (Figure 2). The allometric relationships evaluated for tart cherry and apple individuals sampled

254 are well described by a power law and the empirical estimates of $a$ tend to fall within the bounds

255 of the expectations of FS and overlap with WBE particularly for the relationships defined by

256 diameter and mass. Management of these orchard trees does appear to have some effect on the

257 allometric relationships in the manipulation of the length dimension and the relaxation of

258 biomechanical constraints (Shinozaki et al. 1964). Estimates of $a$ shift significantly for both

259 species between segment, path and subtree branch classifications blurring the scale at which

260 these allometries are consistent within individuals. 
Strong allometric relationships in orchard trees provide support for similar patterns in

262 plant growth, despite different genetic material and management approaches driving growth and

263 architecture among cherry and apple systems. Allometric relationships with multi-dimensional

264 branch dimensions (i.e., surface area and volume) are stronger than relationships with linear

265 dimensions (i.e. length and diameter; Figure 2). This could be because these multi-dimensional

266 branch dimensions better reflect resource transport and environmental exposure (Price et al.

267 preprint; West, Brown and Enquist 1997). For instance, surface area might relate to the number

268 of leaves distributed on a branch, dictating the photosynthetic capacity of that location on the

269 plant (Allen et al. 2005). The volume of a branch might be a better predictor of water use than

270 either length or diameter independently (McCulloh and Sperry 2005). Better performance of

271 multi-dimensional parameters linked to environmental exposure could explain why we find

272 stronger morphological relationships also emerge at the path and subtree level; though, many of

273 the strongest allometric relationships are represented by the highest $r^{2}$ values for all branch

274 classifications (Figures 2B,C,H,I).

275 Existing process-based allometric models, WBE and FS, derive expectations of $a$ for

276 idealized plants. These models provide context for understanding how physiological mechanisms

277 drive the conservation or deviation of plant growth from the idealized expectations (Table 1).

278 The mass $\sim$ diameter relationship is most consistent among species and individuals within our

279 study and is in general agreement with the expected $a$ of the WBE, despite genetic and

280 management differences (Table 2, Figure $2 \mathrm{H}$ ). The diameter $\sim$ volume and diameter $\sim$ area also

281 tend to confer the elastic similarity expectation (Figure 2C,E). The other allometric relationships

282 explored including length dimensions are consistently described by estimated $a$ that shift within 
283 the expectations of idealized plants towards flow similarity and away from the biomechanical

284 constraint of elastic similarity (Figure 2).

285 The shift in $a$ towards flow similarity for allometries including length dimensions may be

286 attributed to the genetic selection for improved production efficiency or to substantial

287 manipulation of tree architecture for commercial fruit production. This study represents two

288 distinct species with clonal genetic material developed through selective breeding. The cherries

289 are clonal scion material with closely related seedling rootstocks, while the apples are clonal

290 scion with five distinct clonal rootstocks that were selected for known differences in tree growth,

291 architecture, and production efficiency (Marini et al. 2014). Manipulation of tree architecture

292 through pruning directly influences length and length-including branch dimensions (i.e., surface

293 area, volume). As much as $25 \%$ of total aboveground biomass is removed annually, which

294 reduces the growth potential of a tree by reducing leaf area and altering the root:shoot balance

295 (Ferree and Schupp, 2003). Localized effects of pruning changes the load-bearing status and

296 growth potential in basal branches. Remaining branches are mainly structurally important

297 branches with wide crotch angles and relatively stubby dimensions suited for bearing a

298 commercial fruit load (Feree and Schupp 2003). Regrowth is invigorated with increased

299 investment in nearby fruit-bearing spurs and lateral branches, potentially driving the shift in $a$

300 towards flow similarity (Grochowska et al. 1984; Fumey et al. 2011). In addition, training of tree

301 architecture with structural supports may influence branch dimensions and localized constraints

302 on branch physiology. Though not statistically significant, we observe that apple is described by

303 an $a$ that shifts slightly more towards flow similarity in relation to tart cherry. The greater shift

304 towards flow similarity in apple is consistent with a relaxation of biomechanical constraints due

305 to direct structural support provided by trellises in the apple system and more intensive 'length- 
306 reducing' pruning cuts. In contrast, the tart cherries are free standing and receive minimal

307 'branch-removing' pruning cuts that may be less of a factor in relaxing biomechanical

308 constraints.

309 The biological process behind strong and consistent allometric relationships is linked to

310 physiological limitations of plant growth in unmanaged plants (McMahon and Kronauer 1976;

311 Niklas and Spatz 2004; Savage et al. 2010). It has remained difficult to disentangle the limiting

312 effects of biomechanical and hydraulic processes, but the insights of the processed-based

313 allometry models provide the opportunity for a first attempt at exploring these constraints in

314 domesticated plants. From this study, it appears that both biomechanical and hydraulic

315 constraints are limiting plant function in orchard systems. Biomechanical constraints may define

316 the diameter of branches while branch length may respond to a relaxation of biomechanical

317 constraints to be hydraulically limited (Figure 2). Biomechanical and hydraulic constraints may

318 also be acting at different levels of branch classification as represented by the shift in estimated $a$

319 among scales. Individual segments are more constrained by load-bearing than what is observed

320 at the path and subtree levels demonstrated by the segment level estimated $a$ shift towards the

321 biomechanically driven elastic similarity expectation while the path and subtree levels estimated

$322 a$ generally shift towards the hydraulically driven flow similarity expectation (Figure 2). This

323 within-canopy shift from elastic similarity to flow similarity may support the concept of

324 'incomplete branch autonomy' by which branches organize themselves independently due to the

325 localized distribution of leaves and the resulting photosynthetic material but ultimately interact

326 within a tree-wide network of nutrient transport and hormone signaling pathways (Marsal et al.

327 2003). The development of theory that disentangles biomechanical and hydraulic constraints 
328 provides the opportunity for further exploration of these physiological mechanisms as they relate

329 to plant allometry.

330 Continued use of a process-based allometric approach in orchard systems may lead to a

331 more general understanding of plant growth that can be linked to physiology and, in the case of

332 orchard trees, can inform management techniques and research programs designed to maintain

333 plant health, increase yields, and reduce resource use (Costes 2004; Niklas 2004; Lauri and

334 Claverie 2008). Describing common allometric relationships and physiological limitations for

335 orchard systems can reveal a boundary where constraints from physics drive plant function more

336 than human intervention. This study finds that allometric relationships are largely invariant

337 between the two orchard species tested, but that the estimated $a$ vary within the idealized

338 expectations of process-based allometric models likely due to the extreme human influence on

339 the plants studied. Both tart cherries and apples were heavily pruned at some stage of their

340 development and received fertilizer, water, and agrochemical applications at levels optimal for

341 reproductive growth. We found that the architecture of the two orchard species are described by

342 allometries indicative of plant growth with reduced biomechanical demands, consistent with the

343 large removal of biomass from the plant during pruning. Despite the large removal of biomass

344 for each of these species, growth following management appears compensatory in such a way

345 that the mass $\sim$ diameter relationship returns to consistent and expected relationships, while

346 length $\sim$ diameter relationships fluctuate potentially according to pruning intensity. Future

347 research might focus on the facets of management that drive allometry the farthest from model

348 expectations or use measurements of body size to standardize treatments that could provide

349 improved analysis of competing orchard systems and varieties. Breeding programs might better 
350 identify varieties that are approaching the yield ceiling while optimizing for resource use

351 efficiency and yield improvements in the varieties with greatest potential for improvement.

352

353

354 Conclusions

355 Our study finds strong allometric relationships in two variably managed orchard species that are

356 broadly consistent among the species. Two process-based models provide context for

357 understanding the potential effects of growing conditions and management on growth and

358 physiology of orchard trees. Agreement with idealized model expectations is limited to the mass

$359 \sim$ diameter relationship of WBE though the empirical estimates of allometric relationships tend

360 to fall within the bounds of the FS towards the flow similarity expectation. This study reveals the

361 potential for continued use of process-based allometry within agricultural systems; however,

362 expectations derived for idealized plants may be insufficient alone for the description of orchard

363 systems due the human manipulation of plants' physiology and growing conditions. Although

364 orchard trees are particularly complex candidates due to genetic, environmental and physical

365 manipulation, process-based allometry may still provide a mechanistic understanding of the

366 effects of management for optimal reproductive growth.

369 Acknowledgements

370 This paper was greatly improved through discussion and review by C. Price, B. Black, K. Mott,

371 J. Reeve, and E. White. Orchard blocks sampled were maintained by T. Lindstrom at the 
372 Kaysville Research Farm, Kaysville, UT. Assistance with field sampling was provided by J.

373 Anderson, J. Shugart, and multiple members of the Weecology lab.

374

375

376

377

378

379

380

381

382

383

384

385

386

387

388

389

390

391

392

393

\section{References}

Allen, M.T., P. Prusinkiewicz and T.M. DeJong. 2005. Using L-systems for modeling sourcesink interactions, architecture and physiology of growing trees: the L-PEACH model. New Phytol. 166:869-880.

Brown, S. 1997. Estimating biomass and biomass change of tropical forests. Food and Agricultural Organization of the United Nations Forestry Paper 134: Rome.

Bucksch, A., A., Atta-Boateng, A.F. Azihou, D. Battogtokh, A. Baumgartner, B.M. Binder, S.A. Braybrook, C. Chang, V. Coneva, T.J. DeWitt, A.G. Fletcher, M.A. Gehan, D.H. DiazMartinez, L. Hong, A.S. Iyer-Pascuzzi, L.L. Klein, S. Leiboff, M. Li, J.P. Lynch, A. Maizel, J.N. Maloof, R.J.C. Markelz, C.C. Martinez, L.A. Miller, W. Mio, W. Palubicki, H. Poorter, C. Pradal, C.A. Price, E. Puttonen, J.B. Reese, R. Rellán-Álvarez, E.P. Spalding, E.E. Sparks, C.N. Topp, J.H. Williams, D.H. Chitwood. Morphological plant modeling: Unleashing geometric and topological potential within the plant sciences. Front. Plant Sci. 8:900.

Budagovsky, V.I. 1974. La sélection des portes-greffes de petite taille de variétés de pommiers résistantes a l'hiver destinées a être cultivées dans les régions centrales de l'union soviétique. In Proc. XIX Int. Hortic. Congr. Ed. R. Antoszwewski, L. Harrison, and C. C. Zych. p. 132-136. 
394 Coomes, D.A., E.R. Lines and R.B. Allen. 2011. Moving on from Metabolic Scaling Theory:

395 Hierarchical models of tree growth and asymmetric competition for light. J. Ecol. 99:748396756.

397 Costes, E. 2004. Integrating knowledge of tree biology and physiology into models of fruit tree 398 development: A review. Acta Hortic. 636:575-589.

399 Deng, J., W. Zuo, Z. Wang, Z. Fan, M. Ji, G. Wang, J. Ran, C. Zhao, J. Liu, K.J. Niklas, S.T. 400 Hammond, and J.H. Brown. 2012. Insights into plant size-density relationships from 401 models and agricultural crops. PNAS 109(22):8600-8605.

402 Enquist, B.J., A.P. Allen, J.H. Brown, J.F. Gillooly|, A.J. Kerkhoff, K.J. Niklas, C.A. Price, G.B. 403 West. 2007. Does the exception prove the rule? Nature. 445: E9-E11.

Fazio, G., H. Aldwinkle, T. Robinson. 2013. Unique characteristics of Geneva ${ }^{\circledR}$ apple 405 rootstocks. New York Fruit Quarterly. 21(2):25-28.

Ferree, D.C. and J.R. Schupp. 2003. Pruning and training physiology. In Apples: Botany, 407 production, and uses. Ed. D.C. Ferree and I.J. Warrington, CAB Intl., MA. 660 p.

Fischer, M. 2001. New dwarfing and semi-dwarfing pillnitz apple and pear rootstocks. Acta Hortic. 557:55-61.

Fumey, D., P.É. Lauri, Y. Guédon, C. Godin and E. Costes. 2011. Effects of pruning on the apple tree: from tree architecture to modeling. Acta Hortic. 903:597-602.

412 Grochowska, M.J., A. Karaszewska, B. Jankowska, J. Maksymiuk and M.W. Williams. 1984. Dormant pruning influences on auxin, gibberellins, and cytokinin levels in apple trees. J. Am. Soc. Hortic. Sci. 109:312-318.

415 Grossman, Y.L. and T.M. DeJong. 1995. Maximum vegetative growth potential and seasonal 416 patterns of resource dynamics during peach growth. Ann. Bot. 76:473-482. 
417 Grossman, Y.L. and T.M. DeJong. 1998. Training and pruning system effects on vegetative

418 growth potential, light interception, and cropping efficiency in peach trees. J. Am. Soc.

419 Hortic. Sci. 123(6):1058-1064.

420 Hoover, E., S. McKay, A. Telias, D. Bedford. 2011. Degree of dwarfing and productivity of 421 eight apple rootstocks with winter hardy scions. Acta Hortic. 903:295-299.

422 DOI: $10.17660 /$ ActaHortic.2011.903.37

423 Huxley, J.S. and G. Teissier. 1936. Terminology of Relative Growth. Nature 137(3471): 780-81.

424 Kobe, R.K. 2006. Sapling growth as a function of light and landscape-level variation in soil 425 water and foliar N in northern Michigan. Oecologia 147:119-133.

426 Lacointe, A. 2000. Carbon allocation among tree organs: A review of basic processes and 427 representation in functional-structural tree models. Ann. For. Sci. 57:521-533.

428 Lang, G.A. and R.J. Lang. 2008. VCHERRY - An interactive growth, training, and fruiting model to simulate sweet cherry tree development, yield and fruit size. Acta Hort. $803: 235-241$.

Lauri, P.E. and J. Claverie. 2008. Sweet cherry tree architecture, physiology and management: Towards an integrated view. Acta Hortic. 795:605-614.

Lauri, P.E., B. Hucbourg, M. Ramonguilhem, D. Méry. 2011. An architectural-based tree training and pruning - Identification of key features in the apple. Acta Hortic. 903:589596.

Le Roux, X., A. Lacointe, A. Escobar-Gutiérrez, and S. Le Dizès. 2011. Carbon-based models of 437 individual tree growth: A critical appraisal. Ann. For. Sci. 58:469-506. 
438 Maguylo, K., G.A. Lang and R.I. Perry. 2004. Rootstock genotype affects flower distribution and 439 density of 'Hedelfinger' sweet cherry and 'Montmorency' sour cherry. Acta Hortic.

440 636:259-266.

441

442

443

444

445

446

447

448

449

450

451

452

453

454

455

456

457

458

459

460

Marini, R.P., B. Black, R. M. Crassweller, P. A. Domoto, C. Hampson, S. Johnson, K. Kosola, S. Mcartney, J. Masabni, R. Moran, R. P. Quezada, T. Robinson and C.R. Rom. 2009. Performance of 'Golden Delicious' apple on 23 rootstocks at 12 locations: A five-year summary of the 2003 NC-140 Dwarf Rootstock Trial. J. Amer. Pomol. Soc. 63(3):115127.

Marini, R.P., B. Black, R.M. Crassweller, P.A. Domoto, C. Hampson,R. Moran, T. Robinson, M. Stasiak and D. Wolfe. 2014. Performance of 'Golden Delicious' apple on23 rootstocks at eight locations: A ten-year summary of the 2003 NC-140 Dwarf Rootstock Trial. J. Amer. Pomol. Soc. 68(2): 54-68 2014.

Marsal, J., B. Basile, L. Solari and T.M. DeJong. 2003. Influence of branch autonomy on fruit, scaffold, trunk and root growth stage III of peach fruit development. Tree Physiol. $23: 313-323$.

McCulloh, K.A. and J.S. Sperry. 2005. Patterns in hydraulic architecture and their implications for transport efficiency. Tree Physiol. 25:257-267.

McMahon, T.A. and R.E. Kronauer. 1976. Tree structures: Deducing the principal of mechanical design. J. Theor. Biol. 59:443-466.

Murneek, A.E. and J.C. Logan. 1932. Autumnal migration of nitrogen and carbohydrates in the apple tree, with special reference to leaves. MO AES Res. Bull. 171.

Niklas, K.J. 1992. Plant Biomechanics: An engineering approach to plant form and function. The University of Chicago Press. 
461 Niklas, K.J. 1997. The evolutionary biology of plants. The University of Chicago Press

462 Niklas, K.J. 2004. Plant allometry: is there a grand unifying theory? Biol. Rev. 79:871-889.

463 Niklas, K.J. and S.T. Hammond. 2014. Assessing scaling relationships: uses, abuses, and $464 \quad$ alternatives. Int. J. Plant Sci. 175(7):754-763.

465 Niklas, K.J. and V. Kerchner. 1984. Mechanical and photosynthetic constraints on the evolution 466 of plant shape. Paleobiology 10:79-101.

467 Niklas, K.J. and H.C. Spatz. 2004. Growth and hydraulic (not mechanical) constraints govern the 468 scaling of tree height and mass. PNAS 101(44):15661-15663.

469 Pacala, S.W. and D. Tilman. 1994. Limiting similarity in mechanistic and spatial models of plant 470 competition in heterogeneous environments. Am. Nat. 143(2):222-257.

471 Price, C.A., B.J Enquist and V.M. Savage. 2007. A general model for allometric covariation in 472 botanical form and function. PNAS 104(32):13204-13209.

473 Price, C.A. J.F. Gilooly, A.P. Allen, J.S. Weitz, and K.J. Niklas. 2010. The Metabolic Theory of 474 Ecology: Prospects and challenges for plant biology. New Phytol. 188:696-710.

Price, C.A. J.S Weitz, V.M. Savage, J. Stegen, A. Clarke, D.A. Coomes, P.S. Dodds, R.S. Etienne, A.J. Kerkhoff, K. McCulloh, K.J. Niklas, H. Olff and N.G. Swenson. 2012. Testing the metabolic theory of ecology. Ecol. Lett. 15:1465-1474.

Price, C.A. P. Drake, E.J. Veneklaas, and M. Renton. preprint. Flow similarity, stochastic branching, and quarter power scaling in plants. arXiv:1507.07820 [q-bio.QM]

Robinson, T.L. 2007. Recent advances and future directions in orchard planting systems. Acta 481 Hortic. 732:367-381.

Robinson, T.L. and S.A. Hoying. 2004. Performance of elite Cornell Geneva apple rootstocks in 483 long-term orchard trials on growers farms. Acta Hortic. 658:221-229. 
484 Savage, V.M., L.P. Bentley, B.J. Enquist, J.S. Sperry, D.D. Smith, P.B. Reich, and E.I. von

485

486

487

488

489

490

491

492

493

494

495

496

497

498

499

500

501

502

503

504

505

506

Allmen. 2010. Hydraulic trade-offs and space filling enable better predictions of vascular structure and function in plants. PNAS 107(52):22722-22727.

Shinozaki K., K. Yoda, K. Hozumi, T. Kira. 1964. A quantitative analysis of plant form-the Pipe Model Theory: I. Basic analysis. Japanese Journal of Ecology 14:97-105.

Smith, D.D., J.S. Sperry, B.J. Enquist, V.M. Savage, K.A. McCulloh, L.P. Bentley. 2013. Deviation from symmetrically self-similar branching in trees predicts altered hydraulics, mechanics, light interception and metabolic scaling. New Phytol. 201(1):217-229.

Soejima, J., Y. Yoshida, T. Haniuda, H. Bessho, S. Tsuchiya, T. Masuda, S. Komori, T. Sanada, Y. Ito, S. Sadamori and Y. Kashimura. 2010. New dwarfing apple rootstocks 'JM 1', 'JM 7' and 'JM 8'. Bull. Nat. Inst. Fruit Tree Sci. 11:1-16.

Stehr, R. 2007. Fifteen years of experience with different dwarfing apple rootstocks in northern Germany. Acta Hortic. 732:67-77.

Thompson, D.W. 1942. On Growth and Form. Cambridge University Press.

Warton, D.I., I.J. Wright, D.S. Falster and M. Westoby. 2006. Bivariate line-fitting methods for allometry. Biol. Rev. 81:259-291.

West, G.B., J.H. Brown, and B.J. Enquist 1997. A general model for the origin of allometric scaling laws in biology. Science 276(5309):122-126.

Westwood, M.N. and A.N. Roberts. 1970. The relationship between trunk cross-sectional area and weight of apple trees. J. Am. Soc. Hortic. Sci. 95(1):28-30.

\section{Figure Legends}


508 Figure 1. An illustration of the branch-level classifications: segment, path and subtree. The

509 allometric analysis was conducted at segment, path and subtree branch classifications for each

510 relationship.

511

512 Figure 2. Estimates of allometric exponent ( $a$ ) and 95\% confidence intervals for five 24-year-old

513 tart cherry (Prunus cerasus; P. mahaleb) and nineteen 10-year-old 'Golden Delicious' apple

514 (Malus spp.; various rootstocks) for each branch-level classification. Segment level estimates are

515 marked by triangles, path by diamonds, and subtree by squares with tart cherry shaded and apple

516 open symbol. The predicted $a$ from the process-bases models are marked as horizontal lines. A-

517 F) The predicted $a$ from the FS model: elastic similarity is marked by a dashed line and flow

518 similarity by a dot-dash line. G-H) The predicted $a$ from the WBE model is marked by a dashed

519 line. 
Figure 1 (on next page)

An illustration of the branch-level classifications: segment, path and subtree. The allometric analysis was conducted at segment, path and subtree branch classifications for each relationship. 


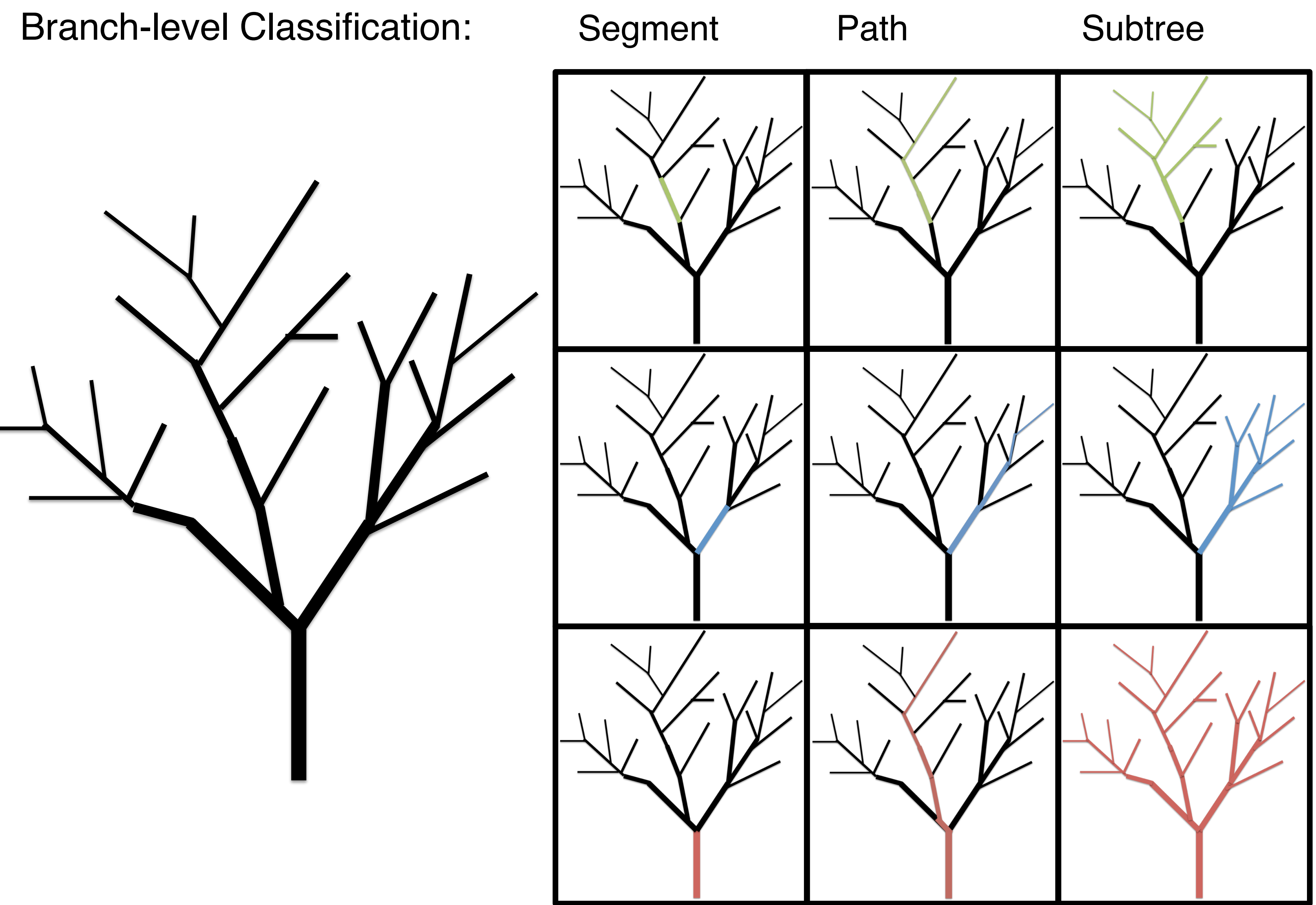


Figure 2 (on next page)

Estimates of allometric exponent (a) and 95\% confidence intervals for five 24-year-old tart cherry and nineteen 10-year-old 'Golden Delicious' apple for each branch-level classification.

Segment level estimates are marked by triangles, path by diamonds, and subtree by squares with tart cherry shaded and apple open symbol. The predicted a from the process-bases models are marked as horizontal lines. (A-F) The predicted a from the FS model: elastic similarity is marked by a dashed line and flow similarity by a dot-dash line. (G-H) The predicted a from the WBE model is marked by a dashed line. 


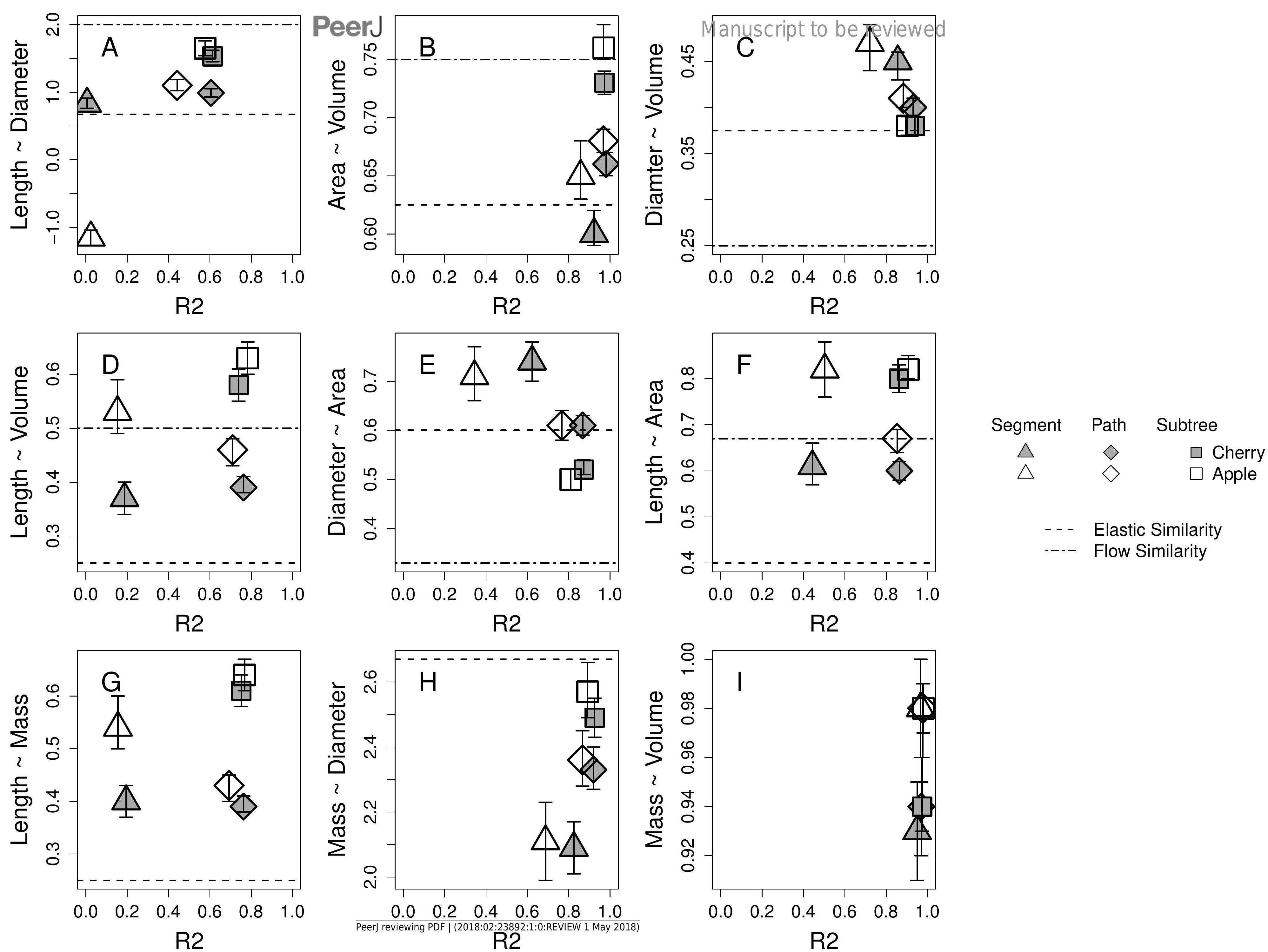




\section{Table $\mathbf{1}$ (on next page)}

Predicted allometric relationships between a.) length, diameter, surface area and volume and b.) length, diameter, and mass

$\mathrm{Y}$ and $\mathrm{X}$ variables as formulated by the Flow Similarity model (FS) and the the West, Brown, Enquist model (WBE) are listed in the first two columns. An expression for each relationship is in the third column, where $\alpha_{\mathrm{F}}$ is the expected exponent for the FS length to diameter prediction and where $\alpha_{M}$ represents the set of expected exponents for the WBE predictions. The following columns represent the predicted exponents. For FS, the predictions are broken down into flow similarity, elastic similarity, and the change in exponent expected going from small to large plants (flow to elastic). This table is modified from Price et al (preprint) with permission. 
1 Table 1-Predicted allometric relationships between a.) length, diameter, surface area and volume

2 as formulated by the Flow Similarity model (FS) and b.) length, diameter, and mass as

3 formulated by the West, Brown, Enquist model (WBE). Y and X variables are listed in the first

4 two columns. An expression for each relationship is in the third column, where $\alpha_{\mathrm{F}}$ is the expected

5 exponent for the FS length to diameter prediction and where $\alpha_{M}$ represents the set of expected

6 exponents for the WBE predictions. The following columns represent the predicted exponents.

7 For FS, the predictions are broken down into flow similarity, elastic similarity, and the change in

8 exponent expected going from small to large plants (flow to elastic). This table is modified from

$9 \quad$ Price et al (preprint) with permission.

10

11 a. FS

$\mid$

\begin{tabular}{|c|c|c|c|c|c|}
\hline Y-variable & $\mathrm{X}$-variable & Expression & $\begin{array}{l}\text { Flow } \\
\text { Similarity }\end{array}$ & $\begin{array}{l}\text { Elastic } \\
\text { Similarity }\end{array}$ & $\begin{array}{l}\text { Changing } \\
\text { Predicted } \\
\text { Exponent }\end{array}$ \\
\hline Length & Diameter & $\mathrm{L}=\mathrm{D}^{\alpha}{ }_{\mathrm{F}}$ & 2 & $2 / 3$ & 2 to $2 / 3$ \\
\hline Surface Area & Volume & $\mathrm{SA}=\mathrm{V}^{\left(\alpha_{\mathrm{F}}+1\right) /\left(\alpha_{\mathrm{F}}+2\right)}$ & $3 / 4$ & $5 / 8$ & $3 / 4$ to $5 / 8$ \\
\hline Diameter & Volume & $\mathrm{D}=\mathrm{V}^{1 /\left(\alpha_{\mathrm{F}}+2\right)}$ & $1 / 4$ & $3 / 8$ & $1 / 4$ to $3 / 8$ \\
\hline Length & Volume & $\left.\mathrm{L}=\mathrm{V}^{\alpha \mathrm{F} /(\alpha} \mathrm{F}_{\mathrm{F}}+2\right)$ & $1 / 2$ & $1 / 4$ & $1 / 2$ to $1 / 4$ \\
\hline Diameter & Surface Area & $\mathrm{D}=\mathrm{SA}^{1 /\left(\alpha_{\mathrm{F}}+1\right)}$ & $1 / 3$ & $3 / 5$ & $1 / 3$ to $3 / 5$ \\
\hline Length & Surface Area & $\mathrm{L}=\mathrm{SA}_{\mathrm{F}}^{\alpha_{\mathrm{F}} /\left(\alpha_{\mathrm{F}}+1\right)}$ & $2 / 3$ & $2 / 5$ & $2 / 3$ to $2 / 5$ \\
\hline
\end{tabular}

12

13

14 b. WBE

\begin{tabular}{llll} 
Y-variable & X-variable & Expression & $\begin{array}{l}\text { Predicted } \\
\text { Exponent }\end{array}$ \\
\hline Length & Diameter & $\mathrm{L}=\mathrm{D}^{\alpha}{ }_{\mathrm{M}}$ & $\geq 1$ to $2 / 3$ \\
Length & Mass & $\mathrm{L}=\mathrm{M}^{\alpha}{ }_{\mathrm{M}}$ & $1 / 4$ \\
Mass & Diameter & $\mathrm{M}=\mathrm{D}^{\alpha}{ }_{\mathrm{M}}$ & $8 / 3$
\end{tabular}




\section{Table 2 (on next page)}

The length $\sim$ diameter and mass $\sim$ diameter branch level allometries for all branches collected for each species: A) cherry, B) apple.

The values reported are the empirically estimated allometric exponent (a) and multiplier (b) or slope and intercept in log-log space, respectively, 95\% confidence intervals (Cl), and $\mathrm{r} 2$ for each branch classification (segment, path, and subtree). 
1 Table 2- The length $\sim$ diameter and mass $\sim$ diameter branch level allometries for all branches

2 collected for each species: a) cherry, b) apple. The values reported are the empirically estimated

3 allometric exponent (a) and multiplier (b) or slope and intercept in log-log space, respectively,

$495 \%$ confidence intervals (CI), and $r^{2}$ for each branch classification (segment, path, and subtree).

a. Cherry

\begin{tabular}{lllllrl} 
& & Estimated $a$ & $95 \%$ CI & \multicolumn{2}{l}{ Estimated $b 95 \%$ CI } & $r^{2}$ \\
\hline Length $\sim$ Diameter & Segment & 0.83 & $0.76-0.91$ & 0.51 & $0.40-0.62$ & 0.006 \\
& Path & 0.99 & $0.93-1.05$ & 0.64 & $0.56-0.73$ & 0.605 \\
& Subtree & 1.53 & $1.45-1.62$ & 0.08 & $-0.05-0.20$ & 0.613 \\
\multirow{3}{*}{ Mass $\sim$ Diameter } & Segment & 2.09 & $2.01-2.17$ & -0.75 & $-0.87--0.64$ & 0.825 \\
& Path & 2.33 & $2.27-2.40$ & -0.90 & $-0.99--0.81$ & 0.920 \\
& Subtree & 2.49 & $2.43-2.55$ & -1.05 & $-1.14--0.96$ & 0.926 \\
\hline
\end{tabular}

b. Apple

\begin{tabular}{lllllll} 
& & Estimated $a$ & $95 \%$ CI & Estimated $b 95 \%$ CI & r2 \\
\hline Length $\sim$ Diameter & Segment & -1.15 & $-1.27--1.04$ & 3.63 & $3.45-3.82$ & 0.024 \\
& Path & 1.10 & $1.02-1.19$ & 0.32 & $0.19-0.46$ & 0.442 \\
& Subtree & 1.65 & $1.54-1.76$ & -0.43 & $-0.61--0.26$ & 0.577 \\
Mass $\sim$ Diameter & Segment & 2.11 & $1.99-2.23$ & -0.67 & $-0.87--0.48$ & 0.688 \\
& Path & 2.36 & $2.28-2.45$ & -0.93 & $-1.07--0.79$ & 0.867 \\
& Subtree & 2.57 & $2.49-2.66$ & -1.21 & $-1.35--1.07$ & 0.892 \\
\hline
\end{tabular}

6 